\title{
Zur ambulanten, psychotherapeutischen Versorgung in den Kantonen Basel-Stadt und Basel-Landschaft
}

\section{Erhebung 1998}

V. Hobi

Korrespondenz:

Prof. Dr. phil. emer. V. Hobi Institut für Klinische Psychologie und Psychotherapie

Psychiatrische Universitätsklinik Wilhelm Klein-Strasse 27

CH-4025 Basel

\section{Einleitung}

Im Sommer 1971 stellte der damalige Leiter des Gesundheitsamtes von Basel-Stadt (Dr. Steiner) eine Kommission zusammen, die den Auftrag erhielt, eine Verordnung (VO) zu entwerfen, nach welcher «nicht-ärztliche» Psychotherapeuten lege artis in die ambulante psychotherapeutische Versorgung psychisch Leidender einbezogen werden könnten. 1975 wurde diese Kommission von Basel-Stadt paritätisch durch Vertreter von Basel-Landschaft ergänzt. 1977 war es dann soweit, dass von beiden Regierungen eine Verordnung für «nicht-ärztliche» Psychotherapie in Kraft gesetzt werden konnte [1]. Die wichtigsten Aspekte der VO 77 seien hier kurz aufgeführt:

§ 8a lic. phil. mit Hauptfach Psychologie oder einer vergleichbaren wissenschaftlichen Ausbildung in Psychologie.

§ 8b Psychopathologie etwa im Rahmen eines Nebenfaches.

§ 8c Ein Jahr klinisches Praktikum unter fachlicher Supervision und

$\S 8 \mathrm{~d}$ eine Ausbildung in einer wissenschaftlich anerkannten, breit anwendbaren Psychotherapiemethode.

§ 4 Bewilligungsinhaber dürfen selbständig psychotherapeutische Behandlungen vornehmen bei Klienten mit Leidenszuständen, bei denen Psychotherapie angezeigt ist, und

§ 5 die Bewilligungsinhaber sind verpflichtet, einen Arzt beizuziehen, wenn der Zustand des Patienten ärztliche Abklärung oder Behandlung erfordert.

Die Beurteilung der Antragsteller nimmt die paritätische Kommission (Psychiater und Psychologen BS und BL) zuhanden der jeweiligen Sanitäts- bzw. Gesundheitsbehörde wahr, die dann die Bewilligungen erteilen. Es wurde damals auch ins Auge gefasst, dass Bewilligungsinhaber/innen ihre Tätigkeit über die Krankenkassen abrechnen konnten.
Die wichtigsten Fragestellungen dieser Untersuchung lauteten u.a.:

- Die Richtung der psychotherapeutischen Ausbildung (Hauptrichtung);

- die diagnostische Zuordnung der Klientel, Vorbehandlung (stationär/ambulant), Art der Zuweisung;

- die Finanzierungsart: Selbstfinanzierung, Krankenkasse, IV;

- Herkunft der Patienten (BS, BL, Region, Ausland);

- Dauer und Ort der Praxis (BS oder BL), Arbeitszeit, Zusammenarbeit (Psychologen/ Psychiater) und das Geschlecht des Therapeuten $(\mathrm{m} / \mathrm{w})$.

Da die gleiche Untersuchung bereits 1982 [2] und 1988 [3] durchgeführt wurde, verzichtete ich auf die Modernisierung des Fragebogens, damit eine gute Vergleichbarkeit des Verlaufs möglich wurde.

Die Beantwortung des Fragebogens konnte anonym durchgeführt werden. Die Auswertung erfolgte weitgehend nach der Anordnung der gestellten Fragen. Die aktualisierte Adressliste stellte uns das Sanitätsdepartement bzw. die Gesundheitsbehörde zur Verfügung. Es wurden nur private Praxen angefragt (also ohne stationäre oder ambulante staatliche Dienste).

\section{Die Ergebnisse}

\section{Zur Rücklaufquote}

Es wurden (vgl. Tab. 1) insgesamt 389 Praxen angeschrieben $(\mathrm{BS}=283$ und $\mathrm{BL}=106)$. Davon haben (vgl. Tab. 2) insgesamt 51\% geantwortet. Bei Post-Wurf-Fragebogen darf dies im fachlich internationalen Vergleich als gut bezeichnet werden. Besonders «antwortfreudig» waren die Psychologen von BL mit 66\%. 
Tabelle 1

Angeschriebene Praxen.

\begin{tabular}{llccccc} 
Index & BS & \multicolumn{3}{c}{ BL } & \multicolumn{3}{c}{ Summe } \\
& N & $\%$ & N & $\%$ & N & $\%$ \\
\hline Psychiater & 145 & 69 & 65 & 31 & 210 & 100 \\
\hline Psychologen & 138 & 77 & 41 & 23 & 179 & 100 \\
\hline Summe & 283 & 73 & 106 & 27 & 389 & 100
\end{tabular}

Tabelle 2

Genauer Rücklauf.

\begin{tabular}{lcccccc} 
Index & BS & \multicolumn{3}{c}{ BL } & \multicolumn{3}{c}{ Summe } \\
& N & $\%$ & N & $\%$ & N & $\%$ \\
\hline Psychiater & 69 & 47 & 32 & 49 & 101 & 48 \\
\hline Psychologen & 71 & 51 & 27 & 66 & 98 & 55 \\
\hline Summe & 140 & 49 & 59 & 56 & 199 & 51
\end{tabular}

Tabelle 3

Hauptgrundausbildung in Psychotherapie.

\begin{tabular}{lcc} 
Index & Psychiater & Psychologe \\
\hline Tiefenpsychologie & $95 \%$ & $54 \%$ \\
\hline Verhaltenstherapie, kognitiv & $0 \%$ & $11 \%$ \\
\hline Gesprächstherapie, Gestalttherapie & $0 \%$ & $28 \%$ \\
\hline Systemtherapie & $3 \%$ & $2 \%$ \\
\hline Körperorientierte Therapie & $2 \%$ & $3 \%$ \\
leer & $0 \%$ & $2 \%$
\end{tabular}

Tabelle 4

«Ich nenne mich ...».

\begin{tabular}{lcc} 
Index & $\begin{array}{l}\text { Psychiater } \\
\text { BS + BL }\end{array}$ & $\begin{array}{l}\text { Psychologe } \\
\text { BS + BL }\end{array}$ \\
\hline Psychoanalytiker & $37 \%$ & $33 \%$ \\
\hline Verhaltenstherapeut, kognitiv & $0 \%$ & $22 \%$ \\
\hline Gesprächstherapeut, Gestalttherapeut & $9 \%$ & $7 \%$ \\
\hline System-, Familientherapeut, Paar-, Gruppentherapeut & $15 \%$ & $5 \%$ \\
\hline Eklektiker & $9 \%$ & $1 \%$ \\
\hline Psychotherapeut & $34 \%$ & $30 \%$ \\
\hline Doppelantworten / leer & $-4 \%$ & $+2 \%$
\end{tabular}

\section{Zur Grundausbildung der Psychologen}

Es hat sich durchgesetzt, dass man national und international von Grundausbildung (eigentliche Berufsausbildung), Weiterbildung (Spezialisierung im angestammten Beruf) und Fortbildung (Vertiefung und Wissenserneuerung und -erweiterung) spricht.
Die VO 77 verlangt für die nicht-ärztlichen Psychotherapeuten eine universitäre Ausbildung in Psychologie. Bei den Bewilligungen 1977 galten Übergangsbestimmungen, so dass es bei der Befragung von 1984 noch 28\% der Bewilligungsinhaber ohne universitäres Studium in Psychologie gab. In der vorliegenden Befragung senkte sich dieser Anteil auf 8\%.

\section{Die Psychotherapeutische Haupt- \\ bzw. Erstausbildung}

Da zwischen BL und BS wenig Unterschiede $\mathrm{zu}$ beobachten waren, können die Ergebnisse bezüglich der Kantone zusammengefasst werden.

Nach wie vor ist im Raum Basel für Psychiater die Tiefenpsychologie die Richtung der ersten Wahl. Das mag sich zukünftig ändern, da auch andere (z.B. die kognitive Verhaltenstherapie) Therapiemethoden ins offizielle Weiterbildungsmodell des FMH-Konzepts für Psychiatrie einbezogen sind.

Die Psychologen zeigen eine deutlichere Diversifikation, insofern neben der Tiefenpsychologie auch die kognitive Verhaltenstherapie (mit 11\%) und zwei klassische humanistische Richtungen (Gesprächstherapie und Gestalt) mit $28 \%$ figurieren.

\section{Die Psychotherapierichtung bezüglich der Anwendung}

Mit Ausnahme der kognitiven VT identifizieren sich (Ergebnis auf die Frage «Ich nenne mich ...») Psychiater und Psychologen recht einheitlich (vgl. Tab. 4).

Hinsichtlich der hauptsächlich in der Praxis zur Anwendung gelangenden Methoden stellen wir bei den Psychiatern wieder den Schwerpunkt in der Analyse bzw. dem psychoanalytisch orientierten Gespräch mit $83 \%$ fest. Bei den Psychologen, neben 55\% mit Psychoanalyse und psychoanalytischem Gespräch auch einen weiteren zusätzlichen Schwerpunkt in VT und GT mit 36\%.

Somit therapieren die Psychiater entsprechend ihrer Ausbildung mehrheitlich tiefenpsychologisch denkend und handelnd; die Psychologen dagegen psychoanalytisch, behavioristisch und nach humanistischer Richtung. Beide Gruppen zeigen aber auch einen mehr oder weniger deutlichen eklektizistisch, polyvalenten Ansatz.

Nur an die Psychologen wurde die Frage zum Verhältnis von Einzel-, Paar- und Gruppentherapie gestellt. Es ist zu beobachten, dass 84\% ihrer Therapieaktivitäten Einzel-, 10\% Paar- und lediglich $6 \%$ aus Gruppentherapie besteht. 
Tabelle 5

Unterschiedliche Tätigkeiten.

$\begin{array}{lcc}\text { Index in \% } & \begin{array}{l}\text { Psychiater } \\ \text { BS + BL }\end{array} & \begin{array}{l}\text { Psychologe } \\ \text { BS + BL }\end{array} \\ \text { Beratung } & 7 & 8 \\ \text { Diagnostik / Gutachten } & 8 & 4 \\ \text { Psychotherapie } & 50 & 77 \\ \text { Psychotherapie und Psychopharmakologie } & 27 & 15 \\ \text { Ausbildung } & 8 & 11\end{array}$

Tabelle 6

Die diagnostische Zuordnung der Klientel.

\begin{tabular}{lll} 
Index (Rangfolge 1.-8.) & $\begin{array}{l}\text { Psychiater } \\
\text { BS + BL }\end{array}$ & $\begin{array}{l}\text { Psychologe } \\
\text { BS + BL }\end{array}$ \\
\hline Entwicklungs- und Reifekrisen & 5. & 3. \\
\hline Neurosen & 2. & 2. \\
\hline Psychosomatik & 4. & 3. \\
\hline Depression, endogen & 4. & 6. \\
\hline Depression, psychogen & 3. & 3. \\
\hline Sucht & 5. & 6. \\
\hline Psychosen & 5. & 6. \\
\hline Psychogeriatrie & 7. & 8.
\end{tabular}

Erläuterung: 1. Rang = hauptsächlich; 8 . Rang selten.

Tabelle 7

Frage nach dem Krankheitswert der Störung.

\begin{tabular}{lcc} 
Index in \% & $\begin{array}{l}\text { Psychiater } \\
\text { BS + BL }\end{array}$ & $\begin{array}{l}\text { Psychologe } \\
\text { BS + BL }\end{array}$ \\
\hline Patienten mit Krankheitswert & 91 & 81 \\
\hline Ohne Krankheitswert & 9 & 19 \\
\hline KK erforderlich & 91 & 81 \\
\hline KK nicht erforderlich & 9 & 19
\end{tabular}

\section{Diversifikation der Praxistätigkeit}

Es dürfte bekannt sein, dass nur wenige der in der privaten Praxis Tätigen sich lediglich psychotherapeutisch betätigen. Im Hinblick auf die ambivalente Versorgungsdichte schien uns die Erfassung dieses Aspektes wichtig.

Aus Tabelle 5 wird ersichtlich, dass von den zwei Berufsgruppen beinahe zu gleichen Teilen Psychotherapie (plus Psychopharmakotherapie des Psychiaters) durchgeführt wird. Dabei fällt auf, dass immerhin 15 \% (vgl. Tab. 5) der Psychologen bei ihrer Psychotherapie Patienten behandeln, die vom Psychiater separat in pharmakotherapeutischer Behandlung stehen. Mit Aus- nahme der Begutachtung (und Diagnostik), bei welcher die Psychiater doppelt so stark beteiligt sind (im Unterschied $\mathrm{zu}$ den Psychologen), arbeiten die zwei Berufsgruppen doch recht einheitlich.

Es lohnt sich, diesen wichtigen Aspekt nochmals hervorzuheben:

- $50 \%$ der Patienten beim Psychiater werden «nur» psychotherapeutisch behandelt;

- $\quad 27 \%$ der Patienten werden beim Psychiater sowohl psychopharmakologisch als auch psychotherapeutisch behandelt;

- $52 \%$ der Patienten beim Psychologen werden «nur» psychotherapeutisch behandelt und

- $15 \%$ der Patienten, die beim Psychologen psychotherapeutisch behandelt werden, werden auch vom Psychiater psychopharmakologisch behandelt.

Damit wird deutlich, dass die Psychologen mit nur einer Differenz von 12\% ausgesprochen psychiatrienahe behandeln.

\section{Die diagnostische Zuordnung der Patienten}

Zur Stütze bzw. Differenzierung der vorherigen Aussage sollten die nächsten Fragen dienen.

Die veraltete, wenig differenzierte Diagnoseeinteilung ist dadurch begründet, dass der Fragebogen von 1982 wegen der Vergleichbarkeit nicht an die modernen Diagnostika angepasst werden sollte.

Hier zeigt sich, dass Psychiater und Psychologen ein recht heterogenes Krankengut behandeln. Lediglich «Entwicklungs- und Reifekrisen» dominieren beim Psychologen mit der Differenz von 2 Rängen und bei den Psychiatern in bezug auf die endogene Depression ebenfalls mit 2 Rängen. Ansonsten besteht eine relative Einheitlichkeit.

Ebenso auffallend ist, dass die überwiegende Mehrheit der Patienten des Psychiaters (71\%) und des Psychologen (66\%) beim Erstkontakt nicht hospitalisiert und auch nicht «vorbehandelt» war. Dagegen sind beim Psychologen 21\% der Patienten ambulant (beim Psychiater 0\%) vorbehandelt worden. Beim Psychiater waren $21 \%$ vorgängig wenigstens $1 \mathrm{mal}$ hospitalisiert und beim Psychologen nur 11\%.

Dies gibt in Verbindung mit Tabelle 6 doch den Hinweis, dass die schweren Störungen (psychogeriatrische Patienten, endogene Depressionen, Psychosen und Sucht) verständlicherweise eher beim Psychiater behandelt werden. Dennoch bleibt festzuhalten, dass Neurosen für beide Psychotherapeutengruppen im gleichen Rang (Rang 2) zahlenmässig insgesamt deutlich überwiegen. 
Tabelle 8

Praxisdauer der Psychiater und Psychologen von BS und BL.

\begin{tabular}{|c|c|c|c|c|}
\hline \multirow[t]{2}{*}{ Index in \% und Jahren } & \multicolumn{2}{|l|}{ Psychiater } & \multicolumn{2}{|c|}{ Psychologen } \\
\hline & BL & BS & BL & BS \\
\hline Seit 3 Jahren & $15 \%$ & $7 \%$ & $33 \%$ & $21 \%$ \\
\hline Seit 8 Jahren & $26 \%$ & $34 \%$ & $26 \%$ & $17 \%$ \\
\hline Total & $41 \%$ & $41 \%$ & $59 \%$ & $38 \%$ \\
\hline Seit 12 Jahren & $44 \%$ & $23 \%$ & $11 \%$ & $20 \%$ \\
\hline Seit 16 Jahren & $0 \%$ & $10 \%$ & $15 \%$ & $16 \%$ \\
\hline Total & $44 \%$ & $33 \%$ & $26 \%$ & $36 \%$ \\
\hline Seit 20 Jahren & $4 \%$ & $15 \%$ & $11 \%$ & $16 \%$ \\
\hline Seit 28-32 Jahren & $10 \%$ & $11 \%$ & $4 \%$ & $10 \%$ \\
\hline \multirow[t]{5}{*}{ Total } & $14 \%$ & $26 \%$ & $15 \%$ & $26 \%$ \\
\hline & $X=11,2 \mathrm{~J}$ & $X=12,6 \mathrm{~J}$ & $\mathrm{X}=8,6 \mathrm{~J}$ & $\mathrm{X}=11,0 \mathrm{~J}$. \\
\hline & $\operatorname{Min}=1$ & $\operatorname{Min}=1$ & $\operatorname{Min}=1$ & $\operatorname{Min}=0$ \\
\hline & $\operatorname{Max}=70$ & $\operatorname{Max}=38$ & $\operatorname{Max}=32$ & $\operatorname{Max}=28$ \\
\hline & $S=13,0$ & $S=8,6$ & $S=7,3$ & $S=7,3$ \\
\hline Zunahme der letzten 11 Jahre & $78 \%$ & $60 \%$ & $70 \%$ & $50 \%$ \\
\hline
\end{tabular}

Tabelle 9

Die Entwicklung des Verhältnisses der Praxen BS und BL von 1982 bis 1998 .

\begin{tabular}{lllll} 
Index & 1982 & & 1998 & \\
& BS & BL & BS & BL \\
\hline Psychiater & $86 \%$ & $14 \%$ & $69 \%$ & $31 \%$ \\
\hline Psychologen & $90 \%$ & $10 \%$ & $77 \%$ & $23 \%$ \\
\hline
\end{tabular}

Diese relative Einheitlichkeit der Klientel wird auch in der nächsten Fragestellung zum Thema Krankheitswert und die Notwendigkeit der Finanzierung durch die Krankenkasse (KK) bestätigt, wo wir lediglich eine Abweichung um $10 \%$ «u Gunsten» des Psychiaters feststellen (vgl. Tab. 7).

In Ergänzung zu diesem Abschnitt ist noch festzuhalten, dass die Psychologen 13\% Patienten haben, bei denen sie «Stütztherapie» durchführen.

\section{Zu einigen Ergebnissen im einzelnen}

Die Praxisdauer der einzelnen Psychotherapeuten gibt einen wertvollen Hinweis auf die Zunahme der Praxen in den letzten Jahren (vgl. Tab. 8).

Aus dieser Übersicht sind zwei Aspekte hervorzuheben:

- In den letzten 11 Jahren haben insbesondere die Psychiaterpraxen in BL (um 78\%) und die Psychologenpraxen in BL (um 70\%) zugenommen.
- Mit Ausnahme der Psychiater BL haben beide Berufsgruppen in den letzten 8 Jahren insgesamt die stärkste Zunahme zu verzeichnen (um 40\%), wobei die Psychologen von BL auch in diesem Zeitabschnitt die höchste Zunahme (59\%) aufweisen.

Noch deutlicher wird dieser Aspekt, wenn das Verhältnis der Praxen dieser beiden Halbkantone (vgl. Tab. 9) betrachtet wird.

Auch hier zeigt sich insgesamt eine deutliche Verschiebung des Verhältnisses «zu Gunsten» von BL (vgl. Tab. 9).

Einige Fragen wurden zur Wirkung der VO von 77 gestellt und zur «Antwort» der KK auf diese: «Ist durch die VO 77 die psychotherapeutische Versorgung verbessert worden?».

Darauf antworten die Psychiater von BS + BL mit $48 \%$ «verbessert», mit $25 \%$ «etwas verbessert» und mit $27 \%$ «nicht verbessert».

Auf die Frage der möglichen Zunahme der «freiwilligen» Finanzierung der psychotherapeutischen Arbeit der Psychologen durch die KK antworten diese mit 5\% «zugenommen», mit 71\% «abgenommen» und $24 \%$ antworten nicht.

Die Haltung der KK widerspricht sachlich in verschiedener Hinsicht den Ergebnissen dieser vorliegenden Untersuchung:

- Wir haben gesehen, dass die Klientel des Psychologen mit derjenigen des Psychiaters doch in hohem Masse vergleichbar ist (siehe u.a. Tab. 6 und 7);

- dass immerhin 70\% der Psychiater durch den offiziellen Einbezug der Psychologen (VO 77) die psychotherapeutische Versorgung mit eher verbessert beurteilen (genaue Angabe: $43 \%$ verbessert; $27 \%$ wenig verbessert und $26 \%$ nicht verbessert bei $4 \%$ Enthaltung);

- dass 90\% der Psychiater «selten» (61\%) bis «öfter» (29\%) Patienten an Psychologen und dass $80 \%$ der Psychologen «selten», (63\%) bis «öfters» $(17 \%)$ Patienten an Psychiater überweisen und

- dass insgesamt 35\% der Patienten des Psychologen von Ärzten bzw. von Psychiatern überwiesen werden.

Zum Versorgungsverhältnis BS + BL

Es ist bekannt, dass die Ärzte- bzw. die Psychiaterund Psychologendichte in Städten grösser ist als in ländlichen Gebieten. So sind die Verhältnisse auch in BS und BL (vgl. hier auch Tab. 1). 
Tabelle 10

Herkunft des Patienten und Praxiskanton des Therapeuten.

\begin{tabular}{lccll} 
Index & Psychiater BL & Psychiater BS & Psychologen BL & Psychologen BS \\
\hline Aus BS & $22 \%$ & $57 \%$ & $30 \%$ & $57 \%$ \\
\hline Aus BL & $66 \%$ & $27 \%$ & $52 \%$ & $25 \%$ \\
\hline Region & $9 \%$ & $9 \%$ & $13 \%$ & $12 \%$ \\
\hline Ausland & $2 \%$ & $3 \%$ & $1 \%$ & $6 \%$
\end{tabular}

Tabelle 11

Versorgungsvolumen der Psychotherapeuten pro Woche. Effektive Zahlen und Hochrechnung (vgl. Tab. $1+2$ ).

\begin{tabular}{lcccc}
\hline Index & Praxen & $\begin{array}{l}\text { Patienten } \\
\text { pro Woche }\end{array}$ & Praxen & $\begin{array}{l}\text { Patienten } \\
\text { pro Woche }\end{array}$ \\
\hline Psychiater BS & 69 & 1932 & 145 & 4060 \\
\hline Psychiater BL & 32 & 896 & 65 & 1820 \\
\hline Psychologen BS & 71 & 1065 & 138 & 2070 \\
\hline Psychologen BL & 27 & 405 & 41 & 615 \\
\hline Psychiater und Psychologen BS & 140 & 2997 & 283 & 6130 \\
\hline Psychiater und Psychologen BL & 59 & 1301 & 106 & 2435 \\
\hline Psychologen BS + BL & 98 & 1470 & 179 & 2685 \\
\hline Psychiater BS + BL & 101 & 2828 & 210 & 5880 \\
\hline Psychiater und Psychologen BS + BL & 199 & 4298 & 389 & 8565 \\
\hline
\end{tabular}

Zum Verhältnis Wohnort des Patienten und Praxisstandort des Therapeuten

Wir können Tabelle 10 recht unterschiedlich interpretieren:

- Es zeigt sich hier eine recht gute «grenzüberschreitende» Aktivität.

- Berücksichtigen wir zusätzlich die Zahl der Praxen, so übernehmen zwar die Therapeuten von BL 22\% (beim Psychiater) bzw. 30\% (beim Psychologen) Patienten von BS; demgegenüber aber befinden sich in den Praxen der Therapeuten von BS 27\% (beim Psychiater) und $25 \%$ (beim Psychologen) von BL.

- Erwähnenswert ist auch, dass die Therapeuten von BS 21\% (bzw. 22\% BL) Patienten aus der Regio betreuen.

Einige «gewagte» Hochrechnungen

zur Kapazität der ambulanten Versorgung

in den Privatpraxen von $B S$ und $B L$

Zum Stichwort «gewagt» ist festzuhalten, dass es sich bei einer solchen Studie nicht um harte Daten handelt.

Dennoch interessieren einige konkrete und hochgerechnete Zahlen, die wir dem Leser nicht vorenthalten wollen. Sie sind jedoch mit der notwendigen Vorsicht zu interpretieren.
Von den bisher mitgeteilten Ergebnissen fehlen zur angesprochenen «Hochrechnung» die Resultate zur Patientenzahl, die wöchentlich behandelt wird. Dies sei hier vorgängig nachgeholt.

Die Psychiater BS + BL geben an, dass sie wöchentlich durchschnittlich 28 Patienten behandeln (Maximum 60; Minimum 4). Demgegenüber behandeln die Psychologen $\mathrm{BS}+\mathrm{BL}$ durchschnittlich 15 Patienten (Maximum 35; Minimum 3).

In Tabelle 1 haben wir die Praxenzahl angegeben, die aufgrund der Unterlagen zu Recht angeschrieben werden konnten, und in Tabelle 2 die Rücklaufquote. Darauf beruhen die nachfolgenden Berechnungen.

Sofern wir die «Unschärferelation» berücksichtigen, bedürfen diese Zahlen keiner weiteren Interpretation.

Die grenzüberschreitenden Aktivitäten im Gegenstandsbereich (vgl. Tab. 10) können wir noch etwas verdeutlichen. Wir stellen dies in der folgenden Tabelle zusammen.

Daraus ergibt sich zusammenfassend (wenn wir nur die hochgerechneten Zahlen berücksichtigen), dass den 585 Patienten, die von BS in BL eine Behandlung erhalten, die Zahl von 1615 Patienten gegenübersteht, die von BL in BS (wöchentlich) behandelt werden.

Die obigen Fragen und Ergebnisse zur psychotherapeutischen Versorgung in den beiden Halbkantonen BS + BL soll mit dem Hinweis auf die Therapeutendichte abgeschlossen werden.

- In BL kommen auf 1 Psychiater 3969 und auf 1 Psychologen (Ps.Th.) 6293 Einwohner (E). Für beide Psychotherapeutengruppen ergibt dies ein Verhältnis von 1:2434 E.

- In BS kommen auf 1 Psychiater 1345 und auf einen Psychologen (Ps.Th.) 1413 Einwohner. Für beide Psychotherapeutengruppen ergibt dies ein Verhältnis von 1:689 E.

- Für beide Halbkantone gilt die Psychiaterdichte von 1:2157 E, die Psychologendichte (Ps. Th.) von 1:2531 E, und für beide Psychotherapeutengruppen gilt das Verhältnis 1:1165 E.

Eine Bemerkung zur relativ hohen Praxendichte (Psychologenpraxen BS und BL N = 179 und Psychiaterpraxen $\mathrm{N}=210$ ) darf nicht fehlen. Die Praxendichte ist in den beiden Halbkantonen seit der ersten Erhebung von $1982(\mathrm{~N}=120)$ bis 1998 um 269 Praxen auf $\mathrm{N}=389$ angewachsen. Der Anstieg ist in BL in den letzten 11 Jahren um ca. 20\% höher als in BS (vgl. hier Tab. 8). Gehen wir aber davon aus, dass nach mehr oder weniger gut belegten Untersuchungen [4] von 9,3\% 
Tabelle 12

Die grenzüberschreitenden Aktivitäten zwischen BS und BL (vgl. auch Tab. $1+2$ ).

\begin{tabular}{|c|c|c|}
\hline Index & Praxen (Gesamtwerte) & Praxen (hochgerechnet) \\
\hline Psychiater BS; & $N=1932$ & $N=4060$ \\
\hline davon $27 \%$ Patienten aus BL & $\mathrm{N}=522$ & $N=1096$ \\
\hline Psychologen BS; & $N=1065$ & $N=2070$ \\
\hline davon $25 \%$ Patienten aus BL & $N=266$ & $N=518$ \\
\hline Psychiater BL; & $N=896$ & $N=1920$ \\
\hline davon $22 \%$ Patienten aus BS & $\mathrm{N}=197$ & $N=400$ \\
\hline Psychologen BL; & $N=405$ & $N=615$ \\
\hline davon $30 \%$ Patienten aus BS & $\mathrm{N}=121$ & $N=185$ \\
\hline
\end{tabular}

Tabelle 13

Geschlecht der Therapeuten.

\begin{tabular}{lll} 
& weiblich & männlich \\
\hline Psychiater BS & $33 \%$ & $67 \%$ \\
\hline Psychiater BL & $19 \%$ & $81 \%$ \\
\hline Psychologen BS & $62 \%$ & $38 \%$ \\
\hline Psychologen BL & $51 \%$ & $48 \%$
\end{tabular}

der Bevölkerung gesprochen wird, die behandlungsbedürftig sind, so kann hier noch nicht von einer Überversorgung ausgegangen werden.

Vor der Abschlussfrage zur multivarianten Statistik sei unkommentiert ein Hinweis auf die Geschlechtsverteilung gegeben.

\section{Zur multivarianten Statistik}

Für die Psychiater gilt:

- dass Psychosen, die in der Praxis behandelt werden, vorgängig hospitalisiert waren $(\mathrm{r}=.60)$;

- dass jene, die vornehmlich psychoanalytisch arbeiten, die Verbesserung durch die VO 77 eher negativ beurteilen $(\mathrm{r}=.59)$;

- dass jene, die «viele» Psychosomatiker behandeln, wenig Psychosen behandeln $(r=.57)$ und

- dass jene Patienten, die ein weniger ausgeprägtes psychiatrisches Krankheitsbild zeigen, öfter an Psychologen delegiert werden ( $\mathrm{r}=.57)$.

Für die Psychologen gilt,

- dass die psychosomatisch Arbeitenden wenig psychogeriatrische Patienten haben $(\mathrm{r}=.63)$;

- dass jene, die Neurosen behandeln, vermehrt auch psychosomatisch Kranke in ihrer Praxis haben $(\mathrm{r}=.50)$;

- dass jene, die Patienten mit Psychosen behandeln, diese vermehrt vom Psychiater psychopharmakologisch behandelt werden $(\mathrm{r}=.40)$;
- dass besonders Störungen mit «Krankheitswert» durch die KK finanziert werden $(\mathrm{r}=.31)$.

Alle genannten Korrelationskoeffizienten sind statistisch hoch signifikant.

\section{Zusammenfassung und kurze Diskussion}

Es wird über die dritte Erhebung zur ambulanten (nicht staatlichen) psychotherapeutischen Versorgung in den beiden Halbkantonen BS + BL berichtet. Ausgangspunkt war in allen drei Erhebungen (1982, 1992 und 1998) die 1977 in Kraft getretene Verordnung für nicht-ärztliche Psychotherapeuten. Als wichtigste Beobachtungen können festgehalten werden:

- Als primäre Psychotherapieausbildung dominiert bei den befragten Psychiatern zu 95\% die tiefenpsychologisch-psychoanalytische Richtung. Bei den Psychologen ist dies «nur» bei $54 \%$ der Fall. Bei letzteren sind kognitive Verhaltenstherapie, Gesprächs- und Gestalttherapie mit knapp 40\% recht deutlich vertreten.

- Hinsichtlich der diagnostischen Zuordnung ihrer Patienten ist ein beträchtliches heterogenes Krankengut beobachtbar. Lediglich im Bereich der Entwicklungs- und Reifekrisen (Psychologen) entgegen endogener Depression (Psychiater) ist ein deutlicher Unterschied (2 Ränge Differenz) festzustellen. Beide Therapeutengruppen behandeln in einem hohen Grad Patienten, die Störungen mit Krankheitswert aufweisen (Psychiater zu 91\% und Psychologen zu 81\%).

- Die Psychotherapeuten beider Basel behandeln pro Woche 8565 Patienten.

- Während die Psychotherapeuten von BL wöchentlich 585 Patienten von BS behandeln, behandeln die Psychotherapeuten von BS 1615 von BL.

- Es wird ein erfreuliches gegenseitiges Zusammenarbeiten festgestellt: 90\% der Psychiater überweisen (selten bis öfter) Patienten an Psychologen und vice versa $80 \%$.

- $73 \%$ der Psychiater (BS + BL) beurteilen die Auswirkung der VO von 1977 auf die psychotherapeutische Versorgung positiv.

Somit bestätigt die Untersuchung, dass die VO von 1977 nach gut 20 Jahren einen wichtigen Platz in der Versorgung psychisch Leidender einnimmt, insbesondere für besser bemittelte Selbstzahler.

Problematisch bleibt die Finanzierung durch die Krankenkassen. Zwar brachte das KVG 1996 
eine offizielle Regelung über die sogenannte delegierte Psychotherapie, die auch in der ersten Revision (2000) enthalten blieb. Im TARMED (voraussichtlich 2004) und in der dritten Revision der «Baustelle» KVG (2004/2005) scheint es diesbezüglich nur minimale Präzisierungen zu geben. Das ist insofern bedauerlich, als für jene Patienten, die auf die KK-Finanzierung angewiesen sind, sehr lange Wartezeiten belegt sind. Stichworte wie «die Psychiatrie platzt aus allen Nähten, Fehleinweisungen, Drehtürpsychiatrie» (vgl. [5]) und Diskriminierung psychisch kranker Menschen [6] bleiben leider vorläufig weiterhin bestehen. Überdeutlich ist diese Problematik im Beobachter unter dem Titel «Psychiatrie in Not» formuliert: Überlastete Kliniken, Ärztemangel, schikanöse Krankenkassen, knauserige Kantone. Psychisch kranke Menschen werden zu Patienten zweiter Klasse [7]. Gleiches gilt übrigens für die Kinder- und Jugendpsychotherapie, sofern Vergleiche mit Deutschland gestattet sind (vgl. Pohl [8]). Dies ist um so bedauerlicher, da im grossen Kuchen der Kosten im Gesundheitswesen 1997 «nur» $0,3 \%$ für die Psychotherapie ausgegeben wurde (für Physiotherapie 1,3\%!) (vgl. [9]).

Trotz der eindeutigen sachlich-fachlichen Begründungen dieser empirischen Ergebnisse bleibt vorläufig aber lediglich die Hoffnung, dass sich die Sorge um die Psyche auch im KVG stärker bemerkbar machen möge. Es gibt dafür vielversprechende verbale Hinweise führender Psychiater (vgl. u.a. Hell [10]) und es gibt immer mehr wissenschaftliche Belege, dass die psychologische Psychotherapie die zentralen Gütekriterien des KVG bestens erfüllt, die da sind: Wirtschaftlichkeit, Zweckmässigkeit und Wirksamkeit.

\section{Literatur}

1 Hobi V. Selbständige Berufsausübung der Psychotherapeutischen Verordnung beider Basel vom 22. November 1977. Schweiz Ärztezeitung 1978;59(46):2053-64.

2 Hobi V, Schüpbach M. Erfahrungsbericht über die «Verordnung betreffend die selbständige Berufsausübung der Psychotherapeuten» beider Basel. Schweiz Ärztezeitung 1984;65(28):1368-77.

3 Hobi V. Zur ambulanten psychotherapeutischen Versorgung in den Kantonen Basel-Stadt und Baselland. Schweiz Ärztezeitung 1990;71(36): 1468-78.

4 Wittchen HU. Bedarfsgerechte Versorgung psychischer Störungen. Unveröffentlichtes Manuskript. München: Max-Planck-Institut für Psychiatrie; 2000.

5 Benini F. Die Psychiatrie platzt aus den Nähten. In: NZZ am Sonntag, 14. April 2002, S. 31.

6 Blickenstorfer M, Mäder-Lutz S, Schaub BR. Diskriminierung psychisch kranker Menschen. In: Med. Tribune 2003;36(6):2.

7 Zanoni U. Diskriminierung mit System. In: Beobachter Nr. 3, 7. Februar 2003, S. 16-25.

8 Pohl P. Wer hilft Kindern und Jugendlichen mit psychischen Störungen in dieser Gesellschaft? Psychologie heute 2002;29(10):46-7.

9 Hug J. Das Gesamtsystem steigert die Kosten, nicht einzelne Leistungsträger. In: Pulsus, September 2002, S. 2 und 3.

10 Hell D. Seelenhunger. Der fühlende Mensch und die Wissenschaft vom Leben. Bern: Huber; 2002. 